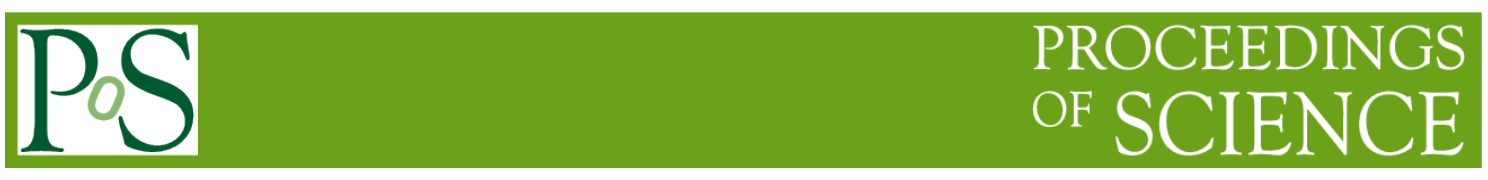

\title{
Nuclear Data for Nuclear Sciences and Applications
}

\section{Paraskevi Dimitriou'}

Nuclear Data Section, International Atomic Energy Agency

Wagramerstrasse 5, A-1400 Vienna, Austria

E-mail: p.dimitriou@iaea.org

The IAEA supports nuclear research activities by providing essential nuclear data and serving as the central agency for the collection and dissemination of data from laboratories worldwide. The EXFOR database contains a compilation of experimental reaction data from all around the world. The Evaluated Nuclear Structure Data File (ENSDF) includes the most extensive and comprehensive set of nuclear structure and decay data evaluations performed by the International Network of Nuclear Structure and Decay Data evaluators, coordinated by the IAEA.

The IAEA has also developed tools for visualization and retrieval of data. The LiveChart application interactively presents nuclide properties taken from ENSDF, while the Isotope Browser provides the same data on a smart phone (Androids and iPhones).

The compilation, evaluation and dissemination of nuclear data are laborious tasks that rely heavily on contributions from experts in both the basic and applied science research communities. Efforts carried out at national and international levels benefit from the coordination provided by the IAEA through its Coordinated Research Projects.

The 26th International Nuclear Physics Conference

11-16 September, 2016

Adelaide, Australia

${ }^{1}$ Speaker 


\section{Introduction}

Nuclear data are essential for many applications including nuclear power, nuclear fusion, medicine, non-destructive testing, and environmental monitoring. Reliable, up-to-date and wellstructured data libraries are indispensable not only for nuclear specialists in the various applications fields, but also for nuclear physics researchers who need the data to improve their knowledge and plan future activities that may lead to new discoveries.

The Nuclear Data Section of the International Atomic Energy Agency develops, maintains and provides free access to nuclear data through user-friendly online databases. These databases provide recommended values for nuclear structure properties such as decay modes, level energies and lifetimes, and radiation properties, as well as for cross sections of particle- and photo-induced nuclear reactions and nuclear fission.

This paper presents an overview of the databases and dissemination tools developed by the IAEA, as well as of the International Network of Nuclear Structure and Decay Data evaluators (NSDD) which is coordinated by the IAEA and is responsible for maintaining the Evaluated Nuclear Structure Data File (ENSDF).

\section{Nuclear Data Activities at the IAEA}

The IAEA supports nuclear research activities in Member States by providing essential nuclear data and serving as the central agency for the collection and dissemination of data from laboratories worldwide. Concerted efforts by the IAEA include coordination of activities in Member States, such as EXFOR and ENSDF, coordinated research project (CRP) implementation, and also staff efforts, which have resulted in data libraries of immense value [1].

The EXFOR database [2] contains a compilation of experimental reaction data from all around the world, and this effort - led by the IAEA - has been very well received, as it provides invaluable data for nuclear calculations and evaluations to researchers in Member States. The EXFOR database covers over 21000 experiments and contains over11.5 million data points. Data have been compiled since 1935 and cover reactions induced by neutrons, charged particles and photons.

Evaluated nuclear data libraries in ENDF format [3] have been produced by many data centres throughout the world. Twenty-six of these libraries can be interrogated and cross-section data plotted. Experimental data from EXFOR can then be added to provide evaluation validation.

The Medical Portal [4] provides a unified view of medical applications, including databases, documents, libraries and ongoing projects. Links to diagnostic and therapeutic radioisotope production, heavy charged particle interactions and phase-space databases are only a mouse click away.

IBANDL [5] and RIPL-3 [6] are examples of two databases resulting from CRPs. Ion Beam Analysis Nuclear Data Library (IBANDL) is a database of experimental and evaluated nuclear cross-sections relevant to ion beam analysis. Reference Input Parameter Library (RIPL3 ) is a library of reference input parameters which are essential ingredients of theoretical modelling codes. By providing a complete set of verified parameters, it is ensured that 
evaluations carried out around the world are compatible and can be produced easily and efficiently.

\section{International Network of Nuclear Structure and Decay Data Evaluators}

Nuclear structure and decay data are important data for a wide range of applications, from the basic nuclear sciences to other fields such as medicine, reactor design and operation, geophysics, environmental sciences, radiation safety and materials sciences. The Evaluated Nuclear Structure Data File (ENSDF) [7] is a collection of recommended data for nuclear properties such as decay modes, level energies and lifetimes, and radiation properties, for all known nuclides. These data are evaluated and maintained by the international network of Nuclear Structure and Decay Data evaluators (NSDD) [8]. They are peer reviewed, comprehensive and available free of charge.

The NSDD network was established in 1974 under the auspices of the IAEA, and includes 16 data centres and over 20 internationally-recognized experts from more than 10 countries. Scientists from five US national laboratories and one university: Argonne National Laboratory, Brookhaven National Laboratory, Lawrence Berkeley National Laboratory, Oak Ridge National Laboratory, Triangle Universities National Laboratory, Michigan State University, together with scientists from Canada, China, Japan, Australia, India, Hungary, Romania, and Russia, evaluate nuclear structure and decay data for all known isotopes on an agreed basis; they also compile the most recent experimental nuclear structure and decay published results in the experimental unevaluated nuclear data list (XUNDL) [9]. The evaluations are also published in the journal Nuclear Data Sheets. The compilation and evaluation work makes extensive use of bibliographic information provided by the online Nuclear Science References (NSR) database [10] which over the years has been developed and extended to provide servicies not only to the NSDD network but the nuclear science community at large. These databases contain these data in computerised format and are available both online and offline. They are managed by the National Nuclear Data Center (NNDC) at Brookhaven National Laboratory.

The ENSDF database draws information on atomic masses ( $Q$ values) from the Atomic Mass Evaluation [11] provided by the Atomic Mass Data Centre (France and China), and evaluators obtain lists of references with data awaiting evaluation from the NSR database, and also benefit from the compilations in the XUNDL database. Other than that, ENSDF is a unique database, as it is comprehensive, continuously updated and serves as the source of data for several derivative, special-purposes databases and products as is illustrated in Figure 1. 


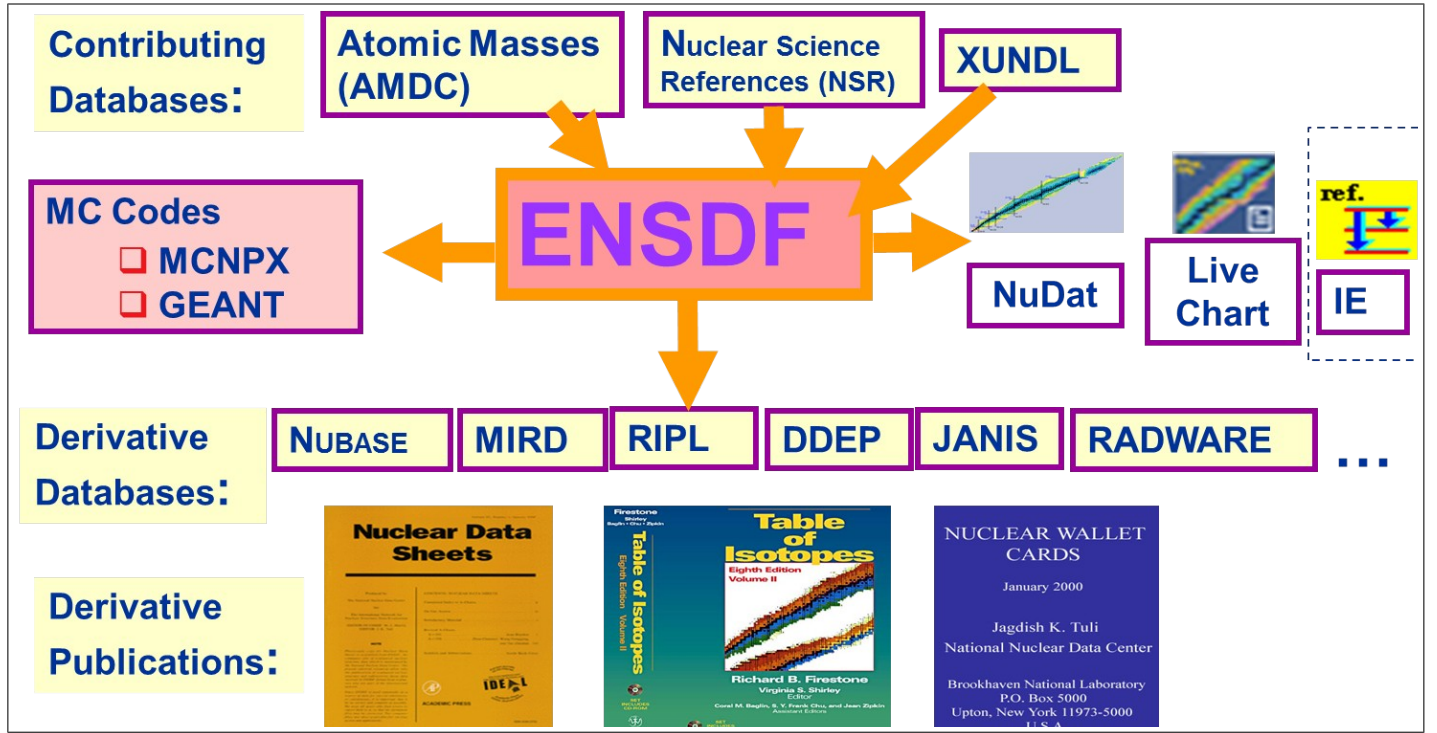

Figure 1. Illustration of the central role of ENSDF in providing evaluated nuclear structure and decay data for a host of other derivative or special-purpose databases.

\section{Dissemination and retrieval tools}

The IAEA has developed a tool (LiveChart) [12] to allow users to interact through a webbased interface with the ENSDF database and perform interactive searches of information from the various structure and decay data in ENSDF and XUNDL, visualize level schemes and decay schemes, and plot certain data across the nuclear chart as a function of mass number (see Figures 2 and 3).

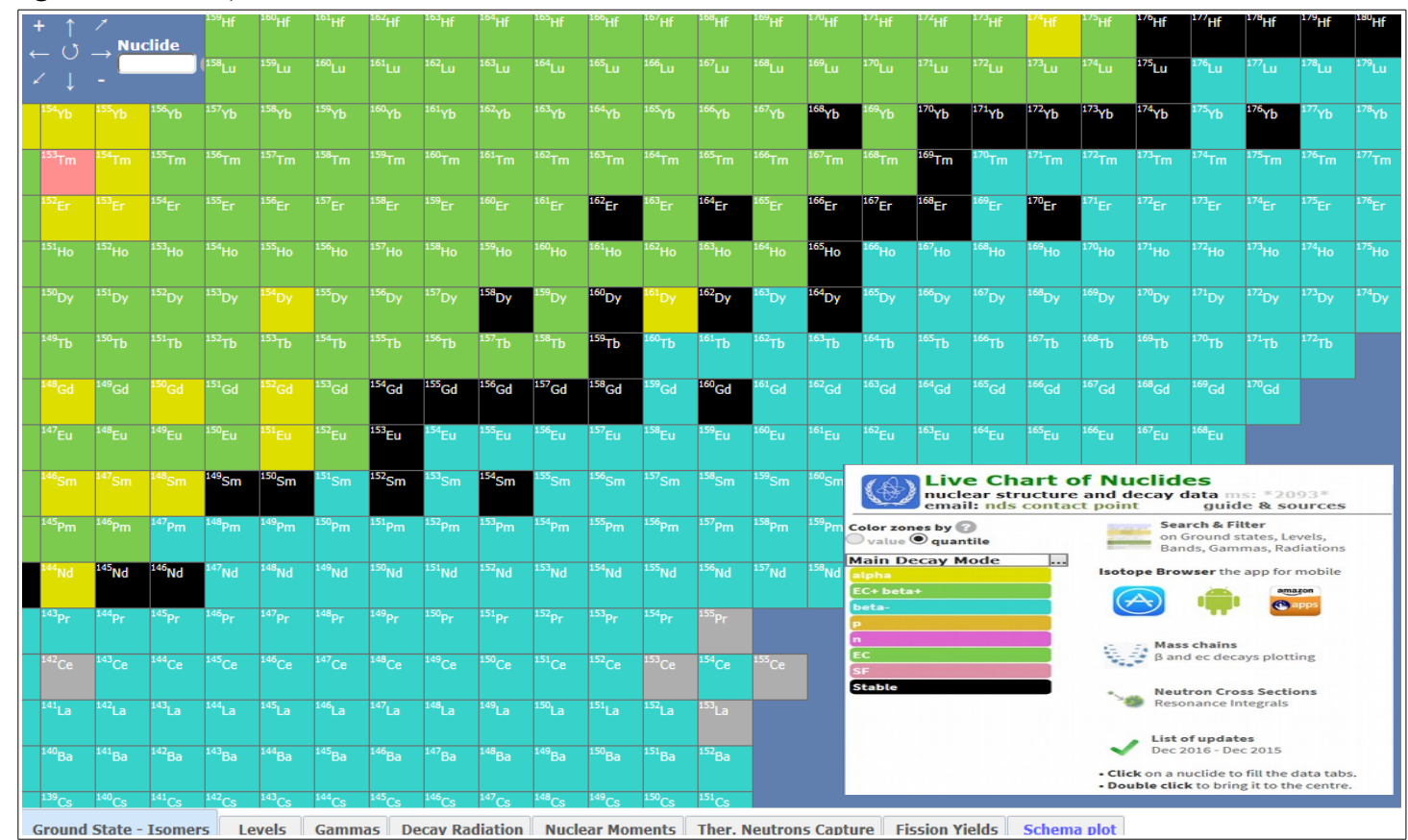

\begin{tabular}{|l|l|l|l|l|l|}
\hline Ground State-Isomers Levels & Gammas Decay Radiation Nuclear Moments Ther. Neutrons Capture & Fission Yields Schema plot \\
\hline
\end{tabular}

Figure 2. The interactive LiveChart application that enables users to find the properties of nuclides, both stable and radioactive, is based on information from the ENSDF database. 


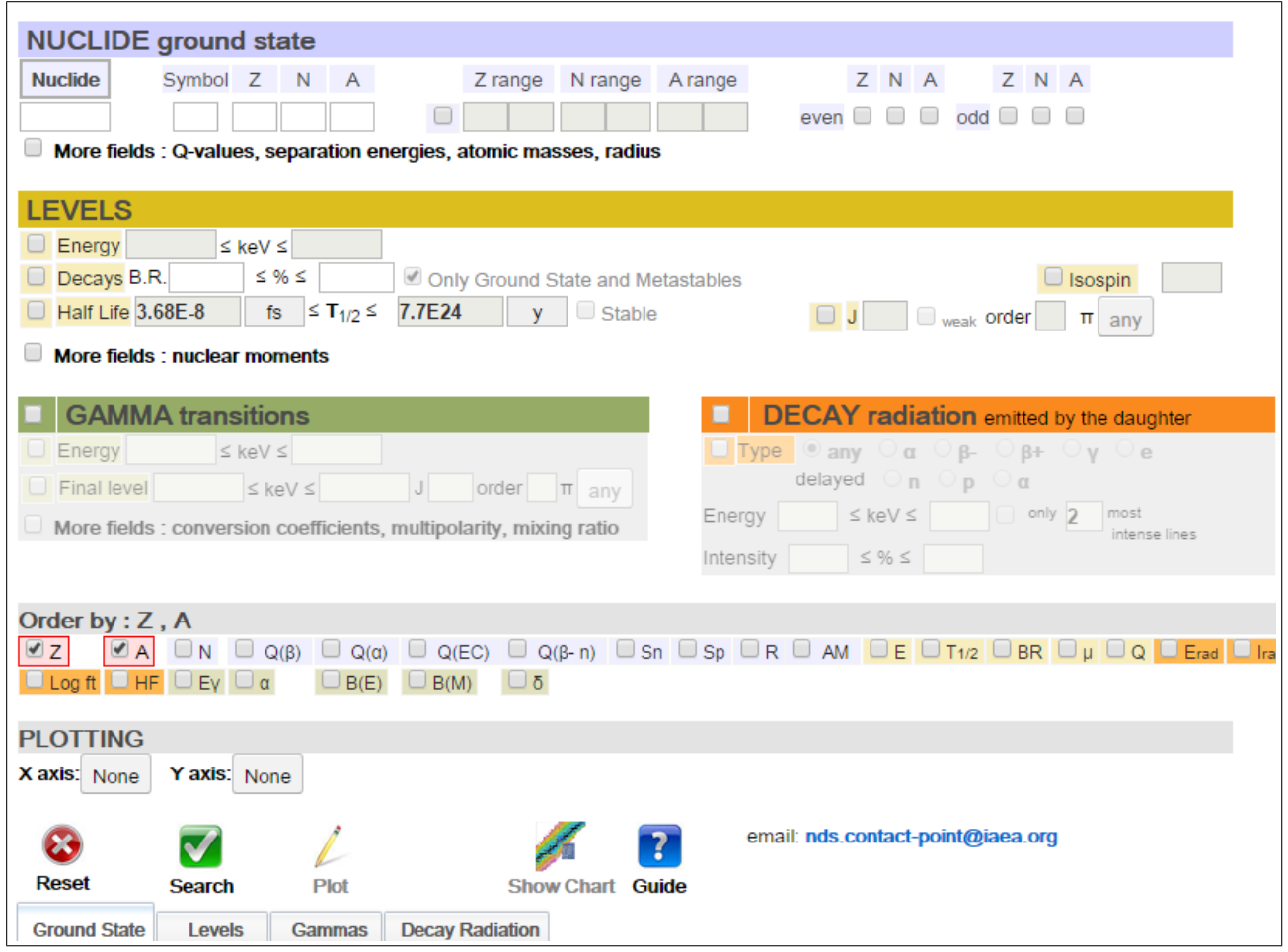

Figure 3. Filtering the ENSDF database throught he Livechart retireval interface.

A further development has been the launching of the Isotope Browser app for mobile devices and tablets. The app gives properties of more than 4000 nuclides and isomers based on information from ENSDF and is the mobile device version of the LiveChart. A Chart of Nuclides, with zooming and tapping enabled, and a Periodic Table of Elements are included to allow easy selection and navigation. Filter criteria on half-life, decay mode, radiation type and energy can be selected. Summary data are presented in a scrolling list, with a details page for each nuclide containing web links to the data sources and further information. Optimal searchand-retrieve performance is achieved with an embedded database, meaning that no network connection is required. The Isotope Browser can be downloaded from the LiveChart web site, or from Google play, Amazon.com and Itunes web sites (see Figure 4).

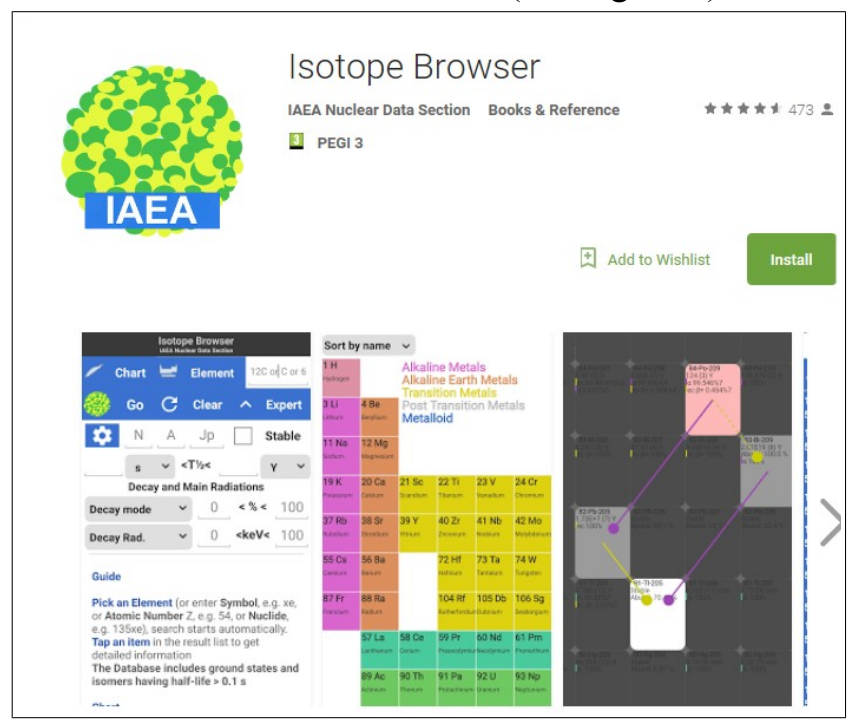

Figure 4. Isotope Browser app for mobile devices. 


\section{Perspectives}

The evaluation and dissemination of nuclear structure and decay data is an international effort coordinated by the IAEA. For many years now, the network has been facing problems in maintaining and updating the ENSDF database with the same regularity as in the past, due mainly to a shortfall in effort coming from Europe, where the retirement of evaluators in the 1990's was not followed by commensurate replacements. An IAEA initiative to bring together nuclear structure specialists from the European Union and Turkey to discuss the current situation with ENSDF specialists in 2008 [13] lead to some positive outcomes: two new european ENSDF Data Centres joined the network (Hungary, Romania), and a European collaborative effort to support ENSDF through the contribution of mass-chain and horizontal evaluations was formed. However, the two data centers only contribute about $10 \%$ of the total effort which is far less than what is expected from a region that is world-known for its technical expertise and output of data from its several top-class, large-scale facilities. The European collaborative effort has made several attempts to secure european funding for ENSDF evaluations in Europe in the period after the IAEA meeting, but without any success at all.

The situation is compounded by the recent retirement of senior evaluators from the US national laboratories, which yet again is not commensurated by an equal number of replacements. There is now an iminent risk of losing the technical expertise in addition to the evaluators. Under these circumstances, the Euorpean nuclear structure community needs to address the following issues:

-is the ENSDF database with recommended nuclear structure data important for their scientific work and for the society at large?

-what would the consequences be (for their current and future projects) if ENSDF were to become out-dated and obsolete?

-is the European community prepared to step up and take action to avert such an unfortunate and disastrous loss of data and expertise?

In an attempt to raise awareness of the situation and the pressing need for action on the part of the European nuclear structure community, the IAEA has liaised with the European NSDD collaboration to promote ENSDF evaluations in Europe and has taken the following actions:

-an abstract and short presentation of the issue was given at the International Conference on Nuclear Data for Science and Technology, 11-16 Sept. 2016, Brugges [14]

-recommendations for support of compilation and evaluation of nuclear structure and decay data in Europe are being introduced in the new NuPECC Long Range Plan [15] which is still in preparation.

Apart from Europe, an effort is also underway to engage other large-scale facilities worldwide, which have a large concentration of active nuclear structure specialists. The RIKEN facility in Japan is one such case, and nuclear structure physicists from RIKEN are already discussing possible schemes for training and collaboration on XUNDL data sets as a first step.

\section{References}

[1] Nuclear Data Services, http://www-nds.iaea.org/ 
[2] Otsuka et al, Nuclear Data Sheets 120 (2014) 272 [http://www-nds.iaea.org/exfor/]

[3] ENDF database, https://www-nds.iaea.org/exfor/endf.htm

[4] Medical Portal, http://www-nds.iaea.org/medportal/

[5] Dimitriou et al, in proceedings of ND2016, 11-16 Sept. 2016, Bruges, EPJ Conferences, to be published [http://www-nds.iaea.org/ibandl/]

[6] Capote et al, Nuclear Data Sheets 110 (2009) 3107 (http://www-nds.iaea.org/RIPL-3/)

[7] ENSDF, http://www.nndc.bnl.gov/ensdf/

[8] International Network of Nuclear Structure and Decay Data Evaluators, http://wwwnds.iaea.org/nsdd/

[9] XUNDL, http://www.nndc.bnl.gov/xundl/

[10] Pritychenko et al, Nucl. Instrum. And Methods A 640 (2011) 2013 [http://www.nndc.bnl.gov/nsr/]

[11] Atomic Mass Data Center, http://amdc.impcas.ac.cn/

[12] LiveChart, http://www-nds.iaea.org/livechart/

[13] Summary Report of ENSDF Technical Meeting, 10-11 November 2008, IAEA, Vienna, $\underline{\text { INDC(NDS) }-0543}$

[14] Negret et al, NSDD Evaluation in Europe, in proceedings of ND2016, 11-16 Sept. 2016, Bruges, EPJ Conferences, to be published

[15] Nuclear Physics European Collaboration Committee (NuPECC) Long Range Plan, http://www.nupecc.org/ 